Chronic norovirus infection in primary immune deficiency disorders : an international case series

Rolfes, M. C.

2019-01

Rolfes , M C , Sriaroon , P , Saldana , B J D , Dvorak , C C , Chapdelaine , H, Ferdman , R M, Chen , K, Jolles , S, Patel , N C , Kim , Y J , Tarrant , T K, Martelius , T, Seppanen , M \& Joshi , A Y 2019 , ' Chronic norovirus infection in primary immune deficiency disorders : an international case series ', Diagnostic Microbiology and Infectious Disease , vol. 93 , no. 1 , pp. 69-73 . https://doi.org/10.1016/j.diagmicrobio.2018.08.002

http://hdl.handle.net/10138/310699

https://doi.org/10.1016/j.diagmicrobio.2018.08.002

publishedVersion

Downloaded from Helda, University of Helsinki institutional repository.

This is an electronic reprint of the original article.

This reprint may differ from the original in pagination and typographic detail.

Please cite the original version. 


\title{
Chronic norovirus infection in primary immune deficiency disorders: an international case series 3 , 许访
}

\author{
M.C. Rolfes ${ }^{\text {a }}$, P. Sriaroon ${ }^{\text {b }}$, B.J. Dávila Saldaña ${ }^{c}$, C.C. Dvorak ${ }^{\text {d }}$, H. Chapdelaine ${ }^{\text {e }}$, R.M. Ferdman ${ }^{\text {f }}$, K. Chen ${ }^{g}$, \\ S. Jolles ${ }^{\text {h }}$, N.C. Patel ${ }^{\text {i }}$, Y.J. Kim ${ }^{\text {j, }}$, T.K. Tarrant ${ }^{\text {k }}$, T Martelius ${ }^{1}$, M. Seppanen ${ }^{\text {l,m }}$, A.Y. Joshi ${ }^{\text {a,n, } *}$ \\ a Mayo Clinic School of Medicine, Rochester, MN \\ ${ }^{\mathrm{b}}$ USF/All Children's Hospital Allergy/Immunology, St. Petersburg, FL \\ c Division of Blood and Marrow Transplantation, Children's National Health System, Washington, DC \\ d Division of Pediatric Allergy, Immunology, and Bone Marrow Transplantation, University of California, San Francisco, CA \\ e Department of Allergy and Immunology, Centre Hospitalier de l'Université de Montréal, Montreal, Quebec, Canada \\ ${ }^{\mathrm{f}}$ Division of Clinical Immunology and Allergy, Children's Hospital Los Angeles, Department of Pediatrics, Keck School of Medicine, University of Southern California, Los Angeles, CA \\ ${ }^{\mathrm{g}}$ Department of Pediatrics, Division of Allergy and Immunology, University of Utah School of Medicine, Salt Lake City, UT \\ ${ }^{\text {h }}$ Immunodeficiency Centre for Wales, University Hospital of Wales, Cardiff, UK \\ ${ }^{i}$ Division of Pediatric Infectious Disease and Immunology, Levine Children's Hospital, Carolinas Medical Center, Charlotte, NC \\ j Division of Infectious Diseases and Immunodeficiency, Department of Pediatrics, Samsung Medical Center, Sungkyunkwan University School of Medicine, Seoul, Republic of Korea \\ k Division of Rheumatology and Immunology, Department of Medicine, Duke University, Durham, NC \\ ${ }^{1}$ Adult Immunodeficiency Unit, Infectious Diseases, Inflammation Center, University of Helsinki and Helsinki University Hospital, Helsinki, Finland \\ ${ }^{m}$ Rare Disease Center, Children's Hospital, University of Helsinki and Helsinki University Hospital, Helsinki, Finland \\ n Division of Pediatric Allergy and Immunology, Department of Pediatric and Adolescent Medicine, Mayo Clinic Children's Center, Rochester, MN
}

\section{A R T I C L E I N F O}

Article history:

Received 5 January 2018

Received in revised form 17 July 2018

Accepted 6 August 2018

Available online 12 August 2018

\section{Keywords:}

Norovirus

Primary immunodeficiency diseases PIDD

CVID, SCID, transplant, immunocompromised

Chronic gastroenteritis

\begin{abstract}
A B S T R A C T
Objective: Predictive factors associated with clinical outcomes of chronic norovirus infection (CNI) in primary immunodeficiency diseases (PIDD) are lacking.

Method: We sought to characterize CNI using a multi-institutional cohort of patients with PIDD and CNI using the Clinical Immunology Society's CIS-PIDD Listserv e-mail group.

Results: Thirty-four subjects ( 21 males and 13 females) were reported from centers across North America, Europe, and Asia. All subjects were receiving high doses (median IgG dose: $1200 \mathrm{mg} / \mathrm{kg} / \mathrm{month}$ ) of supplemental immunoglobulin therapy. Fifty-three percent had a complete absence of B cells (median B-cell count 0; range 0-139 cells $/ \mu \mathrm{L}$ ). Common Variable Immune Deficiency (CVID) subjects manifested a unique phenotype with B-cell lymphopenia, non $\mathrm{O}+$ blood type, and villous atrophy (logistic regression model, $P=0.01$ ). Five subjects died, all of whom had no evidence of villous atrophy.

Conclusion: While Norovirus (NoV) is thought to replicate in B cells, in this PIDD cohort of CNI, B-cell lymphopenia was common, indicating that the presence of B lymphocytes is not essential for CNI.
\end{abstract}

(c) 2018 Published by Elsevier Inc.

\section{Introduction}

Norovirus (NoV) has recently emerged as the most common etiology of acute gastroenteritis in the United States. NoV infections are estimated to cause 19-21 million episodes of gastroenteritis and nearly 400,000 ED visits annually, leading to significant societal and economic

Acronyms: PIDD, Primary Immune Deficiency Disease; CVID, Common Variable Immune Deficiency; NoV, Norovirus; CNI, Chronic Norovirus Infection; IgG, Immunoglobulin G; CID, Combined Immune Deficiency; WAS, Wiskott Aldrich Syndrome; SCID, Severe Combined Immune Deficiency; NK, Natural Killer; RT-qPCR, real-time polymerase chain reaction; BMI, Body Mass Index; GI, Genogroup I; GII, Genogroup II; GI/II, Genogroup I/II.

th Funding source: none.

is is Conflicts of interests: none.

* Corresponding author. Tel.: +1-507-538-0127; fax: +1-507-284-0727.

E-mail address: joshi.avni@mayo.edu (A.Y. Joshi). burden (Hall et al., 2013). With the introduction of the rotavirus vaccine in 2006, NoV has become the leading cause of acute gastroenteritis in US children (Payne et al., 2013) and has emerged as a leading pathogen of gastroenteritis worldwide, with a global prevalence of nearly $20 \%$ in patients with acute gastroenteritis (Ahmed et al., 2014). The virus is transmitted via the fecal-oral route, often through person-to-person contact or through contaminated food/water, and is highly infectious as few viral particles are capable of causing disease (Robilotti et al., 2015; Teunis et al., 2008). NoV infections in healthy individuals typically present with vomiting and abdominal cramps, followed by watery diarrhea for an average duration of 2 to 3 days (Glass et al., 2009), with median duration of viral shedding after experimental human infection of 28 days (range: $13-56$ days).

While NoV infections are typically self-limiting in immunocompetent hosts, NoV is an emerging cause of chronic and life-threatening 
gastroenteritis among vulnerable patient populations, especially in children, the elderly, and immunocompromised hosts (Glass et al., 2009). In particular, NoV has been shown to be one of the most common causes of chronic diarrhea in solid organ transplant patients (Angarone et al., 2016), especially in patients who undergo lung (Blanco et al., 2010; Chagla et al., 2013; Gairard-Dory et al., 2014), kidney (Roos-Weil et al., 2011; Schorn et al., 2010; Westhoff et al., 2009), pancreas (Echenique et al., 2016), or heart transplants (Nilsson et al., 2003). Additional literature has shown chronic NoV infections to be prevalent in pediatric oncology patients (Ludwig et al., 2008; Munir et al., 2014; Simon et al., n.d.) and patients who have had chemotherapy or hematopoietic stem cell transplants (Robles et al., 2012; Roddie et al., 2009; Schwartz et al., 2011). The inability of immunocompromised hosts to adequately clear the virus leads to prolonged viral shedding that can result in months to years of persistent diarrhea, malabsorption, and weight loss (Green, 2014). Treatment of a persistent NoV infection has proven challenging in this subset of patients, contributing to prolonged hospital stays, chronic debilitation, and, in severe cases, mortality (Schwartz et al., 2011).

Despite increasing trends of NoV infections in immunocompromised patients, research investigating NoV infections in patients with immune deficiency, dysregulation, and increased risk for infections due to primary immunodeficiency disorders (PIDDs) is limited (Oksenhendler et al., 2008). In addition to sinopulomonary infections, many subsets of PIDD patients are particularly susceptible to gastrointestinal infections like Giardia lamblia, Cryptosporidium, Helicobacter pylori, cytomegalovirus, and Salmonella (Agarwal and Mayer, 2013; Daniels et al., 2007; Oksenhendler et al., 2008).

Several previous cohort studies have demonstrated NoV infection as the cause of prolonged gastroenteropathies in hospitalized children (Brown et al., 2016; Frange et al., 2012; Xerry et al., 2010) and in adults (Duraisingham et al., 2015; Woodward et al., 2015) with immunodeficiencies. However, limitations exist in these studies, mainly due to the relatively small cohort sizes. A standard treatment approach is also lacking. Furthermore, very few studies to date have systematically assessed predictive factors associated with clinical outcomes in patients with PIDD and NoV. Therefore, in the context of increasing recognition of NoV gastroenteropathies in immunocompromised hosts and limited understanding of predictive factors associated with clinical outcome, we sought to characterize a large multi-institutional cohort of patients with PIDD and CNI.

\section{Methods}

We performed a retrospective review of CNI in PIDD by recruiting centers through the Clinical Immunology Society's CIS-PIDD Listserv e-mail service. Data collected included patient initials, date of birth, sex, weight, height, body mass index as well as white blood cell, absolute lymphocyte, $\mathrm{CD} 3^{+}, \mathrm{CD}^{+}, \mathrm{CD}^{+}, \mathrm{CD} 16^{+} 56^{+}$and $\mathrm{CD} 19^{+}$cell counts, and blood type, primary diagnosis, date of diagnosis, IgG therapy, IgG trough, date of first NoV infection, symptoms, hospitalization and duration, treatment given, date of symptom onset, date of symptom resolution, date of NoV shedding resolution, NoV genotype, endoscopy findings, pathology, coexistent infections, presence of NoV in other sites, coexistent CMV, enterovirus or adenovirus replication, outcomes, and date of last follow-up.

\section{Results}

\section{Overall cohort}

Thirty-four subjects (21 males) were reported from centers across North America, Europe, and Asia (Fig. 1) with median duration of CNI of 1.6 years (range $0.95-2.35$ years). Fifty percent of subjects had common variable immunodeficiency (CVID), 23\% had severe combined immunodeficiency disease (SCID), 12\% had combined immunodeficiency

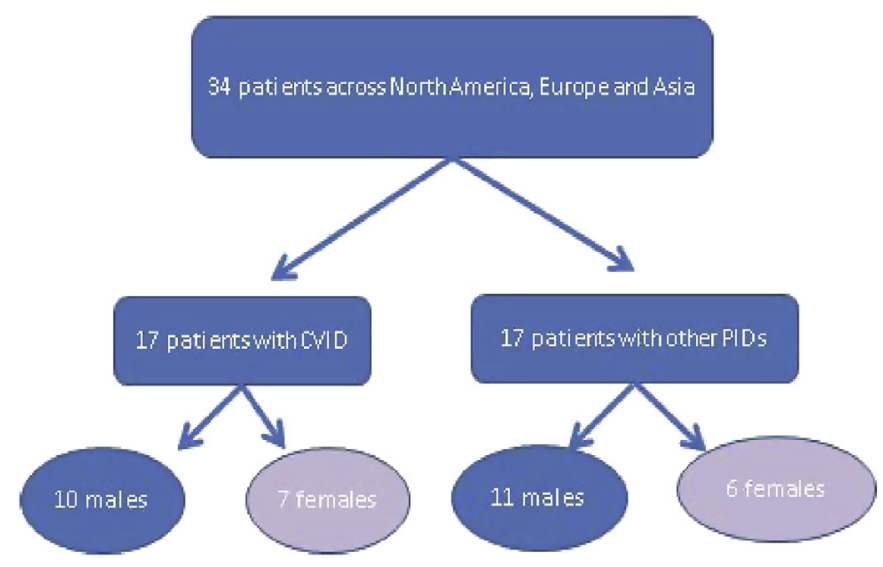

Fig. 1. Study cohort. Demographic distribution of the study cohort.

(CID), and 12\% had Wiskott-Aldrich syndrome (WAS). All subjects were on supplemental immunoglobulin therapy, needing high doses (median IgG dose: $1200 \mathrm{mg} / \mathrm{kg} / \mathrm{month}$ ). Sixty-five percent were hospitalized with CNI (median stay: 47 days; range 7-88 days), and $53 \%$ had a complete absence of B cells (median B-cell count: 0 ; range 0-139 cells $/ \mu \mathrm{L}$ ). T-cell lymphopenia was also seen with a median T-cell count of 650 cells/ $\mu \mathrm{L}$ (range $212-1360$ cells $/ \mu \mathrm{L}$ ).

NoV genotype data were available for 28 out of 34 patients (82\%); genogroup GII was present in 22 subjects (79\%), while GI/GII was identified in 6 patients (21\%). There was a higher likelihood of resolution of CNI with GII (likelihood ratio: 4.6, $P=0.03$ ).

\section{CVID vs. non-CVID}

Table 1 shows the comparison in the baseline characteristics of CVID patients vs. non-CVID patients. CVID patients developed NoV infection at a later age ( 31.23 years vs. 4.5 years, $P<0.001)$. CVID patients also had a higher BMI $\left(20.2 \mathrm{~kg} / \mathrm{m}^{2}\right.$ vs. $\left.16.09 \mathrm{~kg} / \mathrm{m}^{2}, P<0.001\right)$ and were less likely to be blood type $0+$ ( $3 \%$ vs. $76 \%$, OR: 0.15 , CI: $0.04-0.72$, $P=0.01$ ). All patients were on high-dose supplemental IgG therapies. However, CVID patients received relatively lower doses of IgG (1275 mg/kg/month vs. $2666 \mathrm{mg} / \mathrm{kg} / \mathrm{month}, P=0.03)$ and were less likely to be NoV genotype GII (62\% vs. 93\%, OR: 0.11 , CI: $0.01-0.98$, $P=0.045)$. CVID patients also had a longer time of observation with multiple follow-up visits.time (Fig. 2).

Table 1

Basic demographics in CVID and non-CVID CNI cohort.

\begin{tabular}{|c|c|c|c|c|}
\hline & $\begin{array}{l}\text { CVID } \\
(n=17)\end{array}$ & $\begin{array}{l}\text { Non-CVID } \\
(n=17)\end{array}$ & Odds ratio & $P$ value \\
\hline \multicolumn{5}{|l|}{ Sex } \\
\hline Male & $10(59 \%)$ & $11(65 \%)$ & & \\
\hline Female & $7(41 \%)$ & $6(35 \%)$ & & 0.70 \\
\hline \multicolumn{5}{|l|}{ Age of Nov Dx (years) } \\
\hline Mean & 31.23 & 4.5 & & \\
\hline 25-75 percentile & $17.2-49.1$ & $0.6-8.8$ & & \\
\hline Median & 26.5 & 1.53 & & $<0.001^{*}$ \\
\hline \multicolumn{5}{|l|}{ BMI $\left(\mathrm{kg} / \mathrm{m}^{2}\right)$} \\
\hline Mean & 20.2 & 16.1 & & \\
\hline 25-75 percentile & $18.1-21.6$ & $15.03-18.1$ & & \\
\hline Median & 20.4 & 16.09 & & $<0.001^{*}$ \\
\hline Blood type: 0+ & $3(23 \%)$ & $10(76 \%)$ & $0.15(0.03-0.72)$ & $0.01^{*}$ \\
\hline $\begin{array}{l}\text { Supplemental IgG } \\
\text { dosing(mg/kg/month) }\end{array}$ & 1275 & 2666 & & $0.03^{*}$ \\
\hline Hospitalization & $9(56 \%)$ & $12(71 \%)$ & & 0.3 \\
\hline NoV Genotype (GII) & $8(62 \%)$ & $14(93 \%)$ & $0.11(0.01-0.98)$ & $0.045^{*}$ \\
\hline Villous atrophy & $8(47 \%)$ & $4(23 \%)$ & & \\
\hline C. difficile Infection & $5(30 \%)$ & $6(40 \%)$ & $1.61(0.68-3.80)$ & 0.5 \\
\hline
\end{tabular}




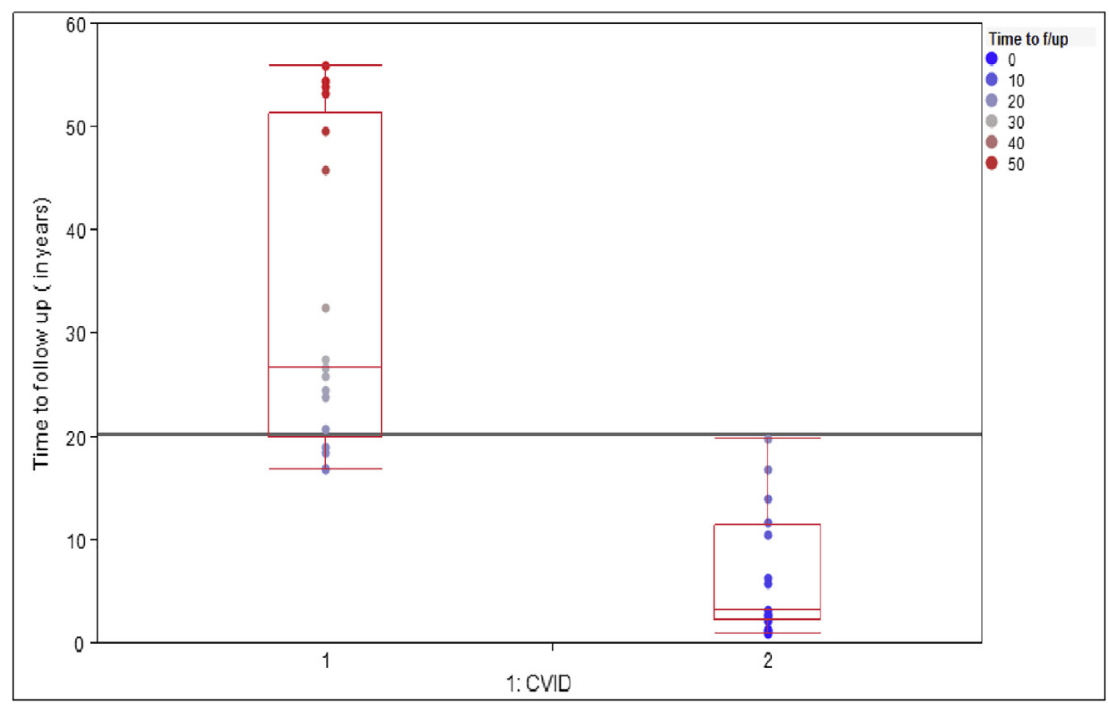

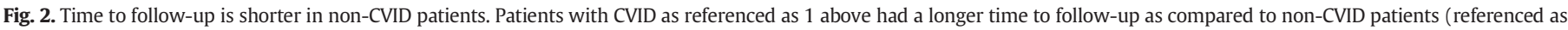
2 above).

Table 2 shows the immune parameters of the cohort. CVID patients vs. non-CVID patients had different immunology profiles. Most notably, patients with CVID had higher total white blood cell counts, absolute lymphocytic counts, CD4 ${ }^{+}$T-cell counts (median 380 cells/ $\mu \mathrm{L}$ vs. 159 cells $/ \mu \mathrm{L}, P=0.03$ ), and $\mathrm{CD} 8^{+}$T-cell counts (median 350 cells $/ \mu \mathrm{L}$ vs. 109 cells $/ \mu \mathrm{L}, P=0.04$ ). CVID patients also had lower $\mathrm{CD} 16^{+} 56^{+} \mathrm{NK}$ cell counts (median 40 cells $/ \mu \mathrm{L}$ vs 205 cells $/ \mu \mathrm{L}, P=0.01$ ). Villous atrophy was seen commonly in CVID patients (Fig. 3) together with increased intraepithelial lymphocytes and absence of plasma cells. Absence of B cells was common in both the CVID and non-CVID subjects, with no significant difference between the 2 subgroups $(P=0.15)$. Concurrent Clostridium difficile infection was reported in 30\% and $40 \%$ of the CVID and non-CVID cohort, respectively.

Resolution of CNI was experienced by 8 (24\%) patients; only 1 of these had CVID. The odds of persistence of CNI in CVID were 11 times higher as compared to non-CVID subjects $(P=0.01)$.

Five subjects died, resulting in a 2-year postdiagnosis survival of 260\% (95\% CI: 55-99\% (Fig. 4). The causes of death included chronic

Table 2

Immune assessment in CVID vs Non-CVID CNI cohort.

\begin{tabular}{|c|c|c|c|c|}
\hline Immune assessment & $\begin{array}{l}\text { CVID } \\
(n=17)\end{array}$ & $\begin{array}{l}\text { Non-CVID } \\
(n=17)\end{array}$ & $\begin{array}{l}\text { Odds } \\
\text { ratio }\end{array}$ & $P$ value \\
\hline $\begin{array}{l}\text { Total WBC count (median) } \\
\left(\times 10^{9} / \mathrm{L}\right)\end{array}$ & 5.9 & 3.9 & & \\
\hline 25-75 percentile & $3.3-9.9$ & $2.8-5.5$ & & 0.09 \\
\hline $\begin{array}{l}\text { Absolute lymphocyte count (ALC) } \\
\text { median }\left(\times 10^{6} / \mathrm{L}\right)\end{array}$ & 1218 & 820 & & \\
\hline $25-75$ percentile & $500-1936$ & $310-1550$ & & 0.5 \\
\hline $\begin{array}{l}\text { CD3 }+ \text { T-cell counts } \\
\text { median }(\text { cells } / \mu \mathrm{L})\end{array}$ & 883 & 329 & & \\
\hline $25-75$ percentile & $224-1894$ & $70-1097$ & & 0.06 \\
\hline $\begin{array}{l}\text { CD4+ T-cell counts } \\
\text { median }(\text { cells } / \mu \mathrm{L})\end{array}$ & 380 & 159 & & \\
\hline 25-75 percentile & $227-700$ & $38-797$ & & $0.03^{*}$ \\
\hline $\begin{array}{l}\text { CD8+ T-cell counts } \\
\text { median }(\text { cells } / \mu \mathrm{L})\end{array}$ & 350 & 109 & & \\
\hline 25-75 percentile & $194-1087$ & $10-655$ & & $0.04^{*}$ \\
\hline $\begin{array}{l}\text { CD16 + 56+ NK-cell counts } \\
\text { median }(\text { cells } / \mu \mathrm{L})\end{array}$ & 40 & 205 & & \\
\hline 25-75 percentile & $17-118$ & $93-448$ & & $0.01^{*}$ \\
\hline $\begin{array}{l}\text { CD19+ cell counts } \\
\text { median }(\text { cells } / \mu \mathrm{L})\end{array}$ & 0 & 0 & & 0.15 \\
\hline $25-75$ percentile & $0-51$ & $0-691$ & & 0.2 \\
\hline
\end{tabular}

lung disease, disseminated mycobacterial avium infection, unresolved diarrhea, and acute aspiration pneumonia. Of these 5 patients, 4 were non-CVID; the odds of survival for CVID subjects were thus nearly 5-fold higher ( $P=0.13$, NS) as compared to the non-CVID subjects. The absence of villous atrophy decreased the chances of survival by nearly 5 -fold $(P=0.03)$ and was a predictor of mortality, but with the current study design, it was difficult to ascertain causality.

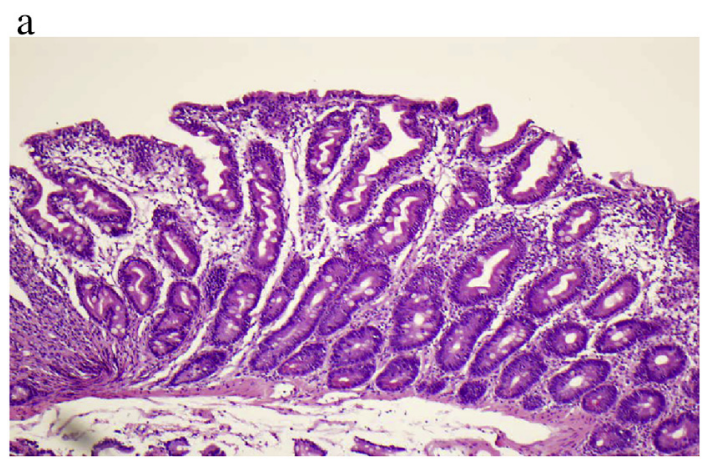

$\mathrm{b}$

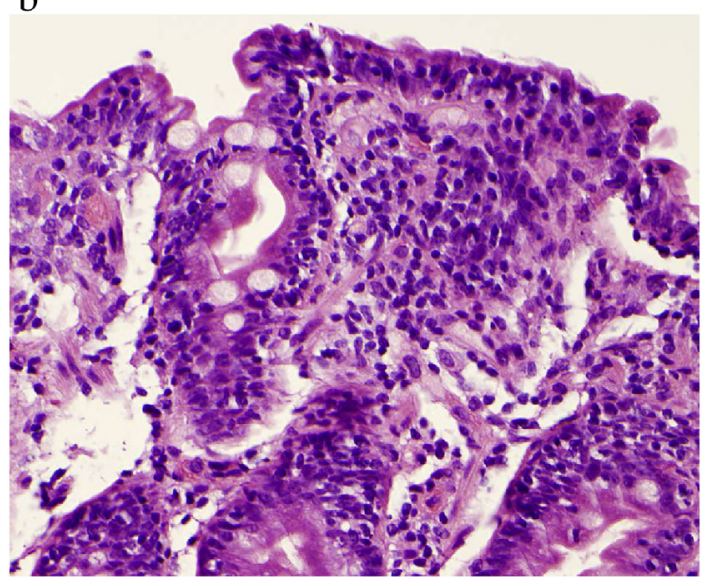

Fig. 3. Histopathology of CNI. a. Low power (20x): CVID with CNI showing villous atrophy and increased intraepithelial lymphocytes. b. High power (40×): CVID with CNI showing rare plasma cells in the lamina propria. 


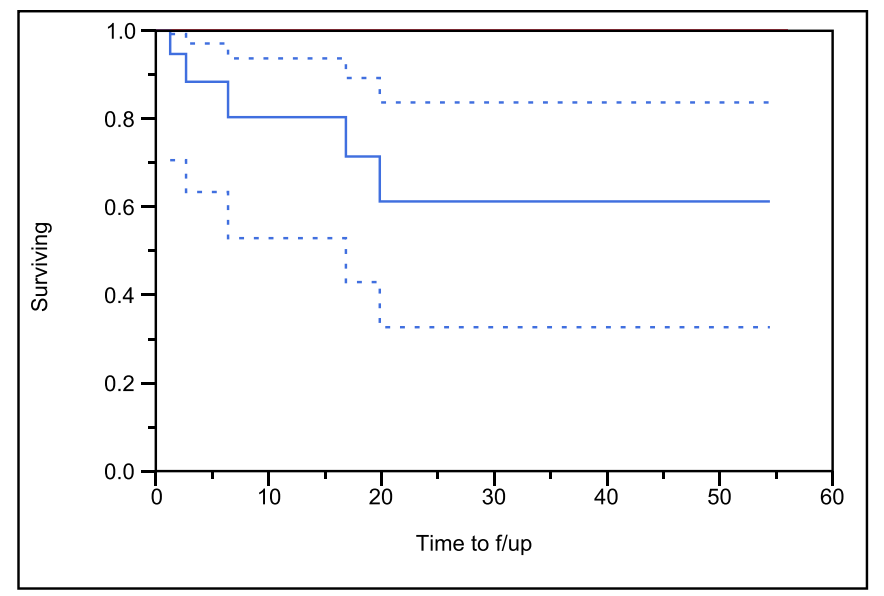

Fig. 4. Kaplan-Meier survival curves. All-cause mortality of the cohort. Blue line showing the absence of villous atrophy patients, $95 \%$ confidence interval ( $\mathrm{CI}$ in dotted lines) as compared to red line showing the cohort with presence of villous atrophy, Wilcoxon test, $P<0.03^{*}$. $Y$ axis shows the probability of surviving, and the $X$ axis is time in months.

\section{Discussion}

To date, this is the first large international multi-institutional study of CNI in PIDDs. In our cohort, we found that B-cell lymphopenia was common, indicating that the presence of $\mathrm{B}$ lymphocytes is not essential for CNI. Death in patients with CNI was seen mostly in the non-CVID cohort and seemed not to be associated with villous atrophy. The majority of our patients experienced severe weight loss and prolonged hospitalizations, leading to chronic debilitation and decreased quality of life. It therefore remains vital for physicians and other health care providers to be aware of increasing rates of NoV infections in PIDD patients, noting that the course of NoV in CVID patients may differ from other PIDDs.

A recent study demonstrated $B$ cells as a target of NoV in mice, indicating that B cells may be an integral part of the establishment of CNI (Jones et al., 2014). However, Brown et al. (2016) demonstrated no difference in NoV between B-cell-positive and B-cell-negative children with SCID. Our study supports findings by Brown et al. by demonstrating that $53 \%$ of patients had both absent B cells and a prolonged course of NoV.

Previous literature has investigated CNI in immunocompromised patients, but research in patients with PIDD is limited. Woodward et al. (2015) analyzed a cohort of 89 adult patients with CVID. Eight (9\%) of these patients had severe enteropathies with significant villous atrophy, and all 8 had fecal excretion of a distinct NoV genotype. Symptoms were prolonged in all cases, with repeatedly positive stool samples over a period of up to 22 months; 2 patients had symptoms for over 6 years. Given that the symptoms resolved with the eventual termination of viral shedding, it was postulated that NoV was causually related to enteropathy in this group of patients, as opposed to being an innocent bystander. Duraisingham et al. (2015) completed a retrospective review of all virology and bacteriology samples from patients attending an immunodeficiency clinic during a 2-year period. Out of 79 patients with severe immunodeficiencies, 6 were found to have NoV based on fecal samples, representing the most common viral infection of the gut. Notably, 3 patients had a persistent infection lasting at least 9, 30, and 16 months, respectively. All 3 patients suffered complications of severe weight loss, 2 patients needed increasing amounts of immunoglobulin therapy, and 1 patient required long-term parenteral nutrition.

Three further cohort studies investigated NoV infections in hospitalized children with immunodeficiencies. Frange et al. (2012) reported that NoV was the most common pathogen detected in fecal samples of children hospitalized for inherited immunodeficiencies, affecting 11 of 62 children. Fecal excretion of NoV was also prolonged, with 57.1\% of fecal samples still positive after a median of 9.5 months of followup (Frange et al., 2012). Brown et al. (2016) supported these results, demonstrating the presence of NoV in fecal samples in 11 of 18 children hospitalized with SCID. Finally, in a study monitoring for contamination in a pediatric immunodeficiency unit in London, UK, Xerry et al. (2010) found $80 \%$ of environmental swabs to be positive for NoV, with subsequent transmission to 2 patients who consequently suffered prolonged gastroenteritis.

Taken together, the above studies demonstrate the presence of CNI in patients with PIDD. However, evidence regarding clinical predictors of outcome is lacking. It is clear that there is a wide spectrum of disease course and outcomes. Some immunocompromised patients have a selflimited disease, while others suffer from prolonged excretion of NoV, leading to debilitation and, in worse cases, mortality. In a previous cohort study of 12 patients with CNI following HSCT, 2 patients died: 1 from unrelated complications and 1 from malnutrition (Roddie et al., 2009). In an additional study of 10 patients with CNI after HSCT, 5 patients died: 3 died due to disease progression without gastrointestinal symptoms, and 2 died due to graft-versus-host-related complications (Ueda et al., 2015). Yet additional investigations are lacking in terms of more specific predictors of disease progression and long-term outcomes in patients with PIDDs. In addition to limited evidence of disease course among immunocompromised patients with CNI, previous literature also fails to establish a standard treatment approach for chronic NoV in immunocompromised patients. The genetic diversity and rapid antigenic variation of NoV and complex interactions with variable host environments appear to contribute to the ongoing therapeutic challenge (Robilotti et al., 2015). There is currently no routine antiviral therapy or vaccine available, and treatment regimens are mainly anecdotal (Green, 2014). Adjustments in immunosuppressant medications have been shown to reduce symptoms and facilitate clearance of NoV in some groups of transplant patients (Roddie et al., 2009; Schorn et al., 2010). Oral immunoglobulins have also shown some promise in immunocompromised patients, with 1 study reporting successful treatment of 11 of 12 lung transplant patients and another study reporting a reduction in diarrhea but not a reduction in timeto-symptom resolution (Florescu et al., 2011; Gairard-Dory et al., 2014). Furthermore, Chagla et al. (2013) demonstrated success with enteral immunoglobulin in a single case report of a transplant recipient. All of our patients received supplemental parenteral IgG, needing high doses (median IgG dose: $1200 \mathrm{mg} / \mathrm{kg} /$ month), but impact on CNI progression was unclear.

Additional medication trials have shown variable effectiveness. In a small, double-blinded placebo-controlled trial in immunocompetent patients, nitazoxanide was shown to be an effective option for enteric viruses including NoV, and 1 case study further reported successful treatment in an immunocompromised patient (Rossignol and El-Gohary, 2006; Siddiq et al., 2011). Ribavirin may also be an option, potentially facilitating clearance of NoV infection in some children with PIDD, although other children with NoV have been refractory (Frange et al., 2012; Woodward et al., 2015). Chen et al. (Siddiq et al., 2011) described the novel development and therapeutic potential of NoV specific monoclonal antibodies, though this approach has not yet been implemented in clinical practice. A NoV vaccine is currently under development and has shown proof of efficacy in human challenge studies; however, it will likely be several more years before it will be available (Riddle and Walker, 2016). Furthermore, use of this vaccine will likely have limitations in immunocompromised hosts. In spite of ongoing efforts, there is clear variability in approaches and outcomes, and treatment in this group of patients continues to pose a challenge. More research in terms of treatment and outcomes of CIN is warranted. Extended-release enteric-coated budesonide has shown variable effects in patients with autoimmune enteropathy (Gentile et al., 2012).

Our study has several limitations. First, as a majority of the data was from multiple institutions, we were unable to obtain longitudinal and 
follow-up data. Secondly, this was a heterogeneous group of PIDD patients, and the medical practices may not have been uniform across the different institutions, which may have the potential to affect the overall outcomes of the patients.

Despite these limitations, our study highlights the increasing need for better recognition and clinical management of $\mathrm{CNI}$ in patients with PIDD. We have demonstrated that the clinical course of patients with CVID versus those with other PIDD may differ, mainly in terms of age of onset, average time to follow-up, and BMI. However, despite the specific PIDD, the majority of the patients in our cohort had need for higher dosing of supplemental IgG therapy and had severe weight loss with prolonged hospitalizations, leading to chronic debilitation and decreased quality of life, with 5 cases resulting in mortality. It is therefore increasingly important for physicians and other health care providers to be aware of increasing rates of NoV infections, particularly in immunocompromised patients. NoV can be detected in a patient's stool or vomit through real-time polymerase chain reaction (RT-qPCR), a test that is widely available. The currently available PCR is qualitative, and there is a greater need for quantitative PCR to track CNI disease progression and burden. In this regard, physicians should have a lower threshold for NoV screening in patients who present with prolonged symptoms of gastroenteritis. Furthermore, as RT-PCR analysis also allows for genetic characterization of NoV strains, there is potential to conduct more expansive epidemiological studies.

\section{Conclusion}

This is one of the first international multi-institutional studies of CNI in PIDD patients. While NoV is thought to replicate in B cells, in this PIDD cohort of CNI, B-cell lymphopenia was common, indicating that the presence of peripheral B lymphocytes is not essential for CNI. It was also interesting to note that death from CNI was not associated with villous atrophy. Furthermore, the clinical course of $\mathrm{CNI}$ in patients with CVID versus other PIDDs may differ. CNI in PIDD patients is severely debilitating, yet predictive factors for outcomes and effective treatment strategies are still unknown. This case series gives insights into the types of PIDD affected by CNI infection, their immunological phenotypes, and the significant therapeutic challenges which remain for this severely debilitating and costly infection in PIDD.

\section{References}

Agarwal S, Mayer L. Diagnosis and treatment of gastrointestinal disorders in patients with primary immunodeficiency. Clin Gastroenterol Hepatol 2013;11:1050-63.

Ahmed S, et al. Global prevalence of norovirus in cases of gastroenteritis: a systematic review and meta-analysis. Lancet Infect Dis 2014;14:725-30.

Angarone MP, Sheahan A, Kamboj M. Norovirus in transplantation. Curr Infect Dis Rep 2016:18:17.

Blanco N, et al. Chronic norovirus gastroenteritis in a double hematopoietic stem cell and lung transplant recipient. Transpl Infect Dis 2010;13:213-5.

Brown JR, Gilmour K, Breuer J. Norovirus infections occur in B-cell-deficient patients. Clin Infect Dis 2016;62:1136-8.

Chagla Z, Quirt J, Woodward K, Neary J, Rutherford C. Chronic norovirus infection in a transplant patient successfully treated with enterally administered immune globulin. J Clin Virol 2013;58:306-8.
Daniels JA, Lederman HM, Maitra A, Montgomery EA. Gastrointestinal tract pathology in patients with common variable immunodeficiency (CVID): a clinicopathologic study and review. Am J Surg Pathol 2007;31:1800-12.

Duraisingham S, et al. Immune deficiency: changing spectrum of pathogens. Clin Exp Immunol 2015;181:267-74.

Echenique IA, et al. Prolonged norovirus infection after pancreas transplantation: a case report and review of chronic norovirus. Transpl Infect Dis 2016;18:98-104.

Florescu DF, et al. Is there a role for oral human immunoglobulin in the treatment for norovirus enteritis in immunocompromised patients? Pediatr Transplant 2011;15: 718-21.

Frange $\mathrm{P}$, et al. Prevalence and clinical impact of norovirus fecal shedding in children with inherited immune deficiencies. J Infect Dis 2012;206:1269-74.

Gairard-Dory A-CC, et al. Clinical usefulness of oral immunoglobulins in lung transplant recipients with norovirus gastroenteritis: a case series. Transplant Proc 2014;46: 3603-5.

Gentile NM, Murray JA, Pardi DS. Autoimmune enteropathy: a review and update of clinical management. Curr Gastroenterol Rep 2012;14:380-5.

Glass RI, Parashar UD, Estes MK. Norovirus gastroenteritis. N Engl J Med 2009;361: 1776-85.

Green K. Norovirus infection in immunocompromised hosts. Clin Microbiol Infect 2014; 20:717-23.

Hall A, et al. Norovirus disease in the United States. Emerg Infect Dis 2013;19:1198-205.

Jones MK, et al. Enteric bacteria promote human and mouse norovirus infection of B cells. Science 2014;346:755-9.

Ludwig Adams, Laws H-J, Schroten, Tenenbaum. Quantitative detection of norovirus excretion in pediatric patients with cancer and prolonged gastroenteritis and shedding of norovirus. J Med Virol 2008;80:1461-7.

Munir N, et al. Norovirus infection in immunocompromised children and children with hospital-acquired acute gastroenteritis. J Med Virol 2014;86:1203-9.

Nilsson M, et al. Evolution of human calicivirus RNA in vivo: accumulation of mutations in the protruding P2 domain of the capsid leads to structural changes and possibly a new phenotype. J Virol 2003;77:13117-24.

Oksenhendler E, et al. Infections in 252 patients with common variable immunodeficiency. Clin Infect Dis 2008;46:1547-54.

Payne D, et al. Norovirus and medically attended gastroenteritis in U.S. children. N Engl J Med 2013;368:1121-30.

Riddle MS, Walker RI. Status of vaccine research and development for norovirus. Vaccine 2016;34:2895-9.

Robilotti E, Deresinksi S, Pinsky B. Norovirus. Clin Mircobiol Rev 2015;28:134-64.

Robles J, Cheuk D, Ha S, Chiang A, Chan G. Norovirus infection in pediatric hematopoietic stem cell transplantation recipients: incidence, risk factors, and outcome. Biol Blood Marrow Transplant 2012;18:1883-9.

Roddie C, et al. Allogeneic hematopoietic stem cell transplantation and norovirus gastroenteritis: a previously unrecognized cause of morbidity. Clin Infect Dis 2009; 49:1061-8

Roos-Weil D, et al. Impact of norovirus/sapovirus-related diarrhea in renal transplant recipients hospitalized for diarrhea. Transplantation 2011:92:61-9.

Rossignol J, El-Gohary Y. Nitazoxanide in the treatment of viral gastroenteritis: a randomized double-blind placebo-controlled clinical trial. Aliment Pharmacol Ther 2006;24: 1423-30.

Schorn R, et al. Chronic norovirus infection after kidney transplantation: molecular evidence for immune-driven viral evolution. Clin Infect Dis 2010;51:307-14.

Schwartz S, et al. Norovirus gastroenteritis causes severe and lethal complications after chemotherapy and hematopoietic stem cell transplantation. Blood 2011:117:5850-6.

Siddiq DM, Koo HL, Adachi JA, Viola GM. Norovirus gastroenteritis successfully treated with nitazoxanide. J Infect 2011;63:394-7.

Simon A, et al. Norovius outbreak in a pediatric oncology unit. Scand. J. Gastroenterol. 2006;41:693-9.

Teunis P, et al. Norwalk virus: how infectious is it? J Med Virol 2008;80:1468-76.

Ueda $\mathrm{R}$, et al. Characteristics and outcomes of patients diagnosed with norovirus gastroenteritis after allogeneic hematopoietic stem cell transplantation based on immunochromatography. Int J Hematol 2015;102:121-8.

Westhoff TH, et al. Chronic norovirus infection in renal transplant recipients. Nephrol Dial Transplant 2009;24:1051-3.

Woodward JM, et al. The role of chronic norovirus infection in the enteropathy associated with common variable immunodeficiency. Am J Gastroenterol 2015;110:320-7.

Xerry J, Gallimore CI, Cubitt D, Gray JJ. Tracking environmental norovirus contamination in a pediatric primary immunodeficiency unit. J Clin Microbiol 2010;48:2552-6. 